\title{
Interconnected Signatures of Quantum Spin Liquid Physics Across the Barlowite Family of Quantum Magnets
}

\author{
Rebecca Smahaa ${ }^{a}$ Wei He ${ }^{b}$, Jack Jiang ${ }^{c}$, Charles Titus ${ }^{d}$, Jiajia Wen ${ }^{e}$, and Young Lee \\ aStanford University, Stanford, California 94305, USA, rsmaha@stanford.edu \\ bStanford University, Stanford, California 94305, USA, weihe@stanford.edu \\ 'Stanford University, Stanford, California 94305, USA, imiiang@stanford.edu \\ 'Stanford University, Stanford, California 94305, USA, cjtitus@stanford.edu \\ eSLAC National Laboratory, Menlo Park, California 94025, USA, jwen11@stanford.edu \\ 'Stanford University, Stanford, California 94305, USA, youngs|@,stanford.edu
}

Quantum magnets display exotic phases that may be strongly influenced by small differences in structure and composition. The quantum spin liquid (QSL) is an unusual magnetic ground state, characterized by long-range quantum entanglement of the spins but a lack of long-range magnetic order down to $T=0 \mathrm{~K}$.[1] This is believed to be possible in highly frustrated $S=1 / 2$ systems,[2] so materials with a kagome arrangement of $\mathrm{Cu}^{2+}$ ions are prime candidates.

Comprehensive structural (single crystal and powder X-ray diffraction, extended X-ray absorption fine structure, powder neutron diffraction, single crystal neutron scattering) and magnetic measurements were performed on newly synthesized variants of $S=1 / 2$ kagome materials barlowite $\left(\mathrm{Cu}_{4}(\mathrm{OH})_{6} \mathrm{FBr}\right)[3]$ and $\mathrm{Zn}$-substituted barlowite $\left(\mathrm{Cu}_{3-x} \mathrm{Zn}_{\times}(\mathrm{OH})_{6} \mathrm{FBr}\right)[4]$ to address open questions about their crystal and magnetic structures.

In contrast to orthorhombic Pnma barlowite, a novel single crystalline variant of barlowite has been synthesized[5] with a less drastic symmetry lowering to hexagonal $P 6_{3} / \mathrm{m}$. The variants demonstrate a clear structure-properties relationship: the distinct low-temperature structures correlate to different magnetic and thermodynamic properties. While their kagome layers are predominantly unperturbed by these phase transitions, the low-temperature structures differ most in the relative occupation of the interlayer distorted trigonal prismatic $\left(C_{2 v}\right) \mathrm{Cu}^{2+}$ sites: orthorhombic barlowite has significant disordering, while hexagonal barlowite has much less disordering. The higher symmetry results in an overall smaller ordered moment compared to orthorhombic barlowite, moving the hexagonal sample closer to a QSL ground state.

Sizable single crystals of QSL candidate $\mathrm{Zn}$-substituted barlowite $\left(\mathrm{Cu}_{3.44} \mathrm{Zn}_{0.56}(\mathrm{OH})_{6} \mathrm{FBr}\right)$ were successfully grown for the first time, and no structural transition occurs upon cooling to low temperatures. Site-specific anomalous dispersion and EXAFS measurements demonstrate that $\mathrm{Zn}^{2+}$ occupies a perfect trigonal prismatic $\left(D_{3 h}\right)$ interlayer site, and no measurable amount substitutes onto the kagome layer. Significantly, no magnetic transition is observed down to $T=$ $0.1 \mathrm{~K}$, consistent with a QSL ground state. Its magnetic response is compared to polycrystalline $\mathrm{Cu}_{3.05} \mathrm{Zn}_{0.95}(\mathrm{OH})_{6} \mathrm{FBr}$ and herbertsmithite. These samples span a spectrum of quantum spin liquidness, allowing this exotic ground state to be probed systematically.

[1] Norman, M. R. (2016). Rev. Mod. Phys. 88, 041002.

[2] Jiang, H. C. et al. (2008). Phys. Rev. Lett. 101, 117203.

[3] Han, T.-H. et al. (2014). Phys. Rev. Lett. 113, 227203.

[4] Feng, Z. et al. (2018). Phys. Rev. B 98, 155127.

[5] Smaha, R. W. et al. (2018). J. Solid State Chem. 268, 123-129. 